\title{
Desenvolver o Pensamento Crítico através da Pesquisa Guiada e Jigsaw: um estudo exploratório num curso de engenharia
}

\author{
Margarida Carvalho, Caroline Dominguez \\ \& Teresa Morais
}

Resumo:

Perante um mundo de aceleradas mudanças que parecem incontroláveis, a sobrevivência das futuras gerações está a ser desafiada tendo impelido as instituições de ensino superior a atender às recomendações recorrentes de organismos internacionais de educação a incluir o Pensamento Crítico (PC) e as soft skills nos curricula. Este artigo contribui para a investigação empírica sobre estratégias ativas de ensino e aprendizagem que promovam o desenvolvimento do PC. Tendo por base uma intervenção pedagógica semestral numa turma de alunos de primeiro ano do curso de licenciatura de engenharia mecânica, procurou-se saber em que medida uma estratégia pedagógica integrada, que articula a Pesquisa Guiada (PG) e o método de Aprendizagem Cooperativa (AC) 'Jigsaw', pode promover capacidades e disposições de PC. Os resultados obtidos apontam para o potencial desta metodologia na aquisição de conhecimento, no desenvolvimento das capacidades de questionar, analisar, avaliar e sintetizar informação e das disposições de autoconfiança e de curiosidade intelectual.

Palavras-chave:

pensamento crítico; pesquisa guiada; Jigsaw e aprendizagem cooperativa; engenharia; ensino superior. 


\title{
Develop critical thinking through guided inquiry and jigsaw: an exploratory study in an engineering course
}

\begin{abstract}
Faced with a world of accelerating changes that seem uncontrollable, the survival of future generations is being challenged and have pushed higher education institutions to comply with the recurrent recommendations of international education bodies to include Critical Thinking and soft skills in the curricula. This article intends to contribute to empirical research on active strategies of teaching and learning that promote the development of Critical Thinking Based on a biannual pedagogical intervention in a class of first-year students of a mechanical engineering degree course, the objective of this article was to reflect to what extent an integrated pedagogical strategy that articulates Guided inquiry and the method of Cooperative Learning "Jigsaw" can promote capacities and dispositions of critical thinking. The results obtained point out to the potential of this combined strategy in the acquisition of knowledge, in the development of better questioning, analyzing evaluating and synthesizing information and in an improvement of the self-confidence and curiosity dispositions.
\end{abstract}

Keywords: critical thinking; guided inquiry; Jigsawan and cooperative learning; engineering course; higher education.

\section{Développer la pensée critique grâce à la recherche guidée et au jigsaw: une étude exploratoire dans un cours d'ingénierie}

Résumé: Face à un monde qui assiste à une accélération des changements qui semblent incontrôlables, la survie des générations futures est mise au défi et fait pression sur les établissements d'enseignement supérieur pour qu'ils suivent les recommandations récurrentes des organismes internationaux d'éducation d'inclure les compétences de la Pensée Critique (PC) et les soft skills dans les programmes d'enseignement. Cet article vise à contribuer à la recherche empirique sur les stratégies actives d'enseignement et d'apprentissage qui favorisent le développement de la PC. Ayant comme base une intervention pédagogique semestrielle dans une classe d'étudiants de première année du cours licence en Ingénierie mécanique, nous avons cherché à savoir dans quelle mesure une stratégie pédagogique intégrée qui articule la Recherche guidée et la méthode d'Apprentissage Cooperative "Jigsaw" peut favoriser les capacités et les dispositions de la PC. Les résultats obtenus soulignent le potentiel de cette stratégie combinée dans l'acquisition de connaissances et dans le développement des capacités à mieux interroger, analyser, évaluer et synthétiser des informations ainsi que les dispositions telles que l'auto confiance et la curiosité.

Mots clés: pensée critique; recherche guidée; Jigsaw et apprentissage coopératif; ingénierie; enseignement supérieur.

\section{Desarrollar el pensamiento crítico a través de la investigación guiada y jigsaw: un estudio exploratorio en un curso de ingeniería}

Resumen: Ante un mundo de acelerados cambios que parecen incontrolables, la supervivencia de las futuras generaciones está siendo desafiada impulsionando las instituciones de enseñanza superior a atender a las recomendaciones recurrentes de organismos internacionales de educación en incluir el Pensamiento Crítico (PC) y las soft skills en los currículos. Este artículo pretende contribuir a la investigación empírica sobre estrategias activas de enseñanza y aprendizaje que promueven el desarrollo del PC. Con base en una intervención pedagógica semestral en una clase de alumnos de primer año del curso de licenciatura de ingeniería mecánica, se buscó saber en qué medida una estrategia pedagógica integrada que articula la Investigación guiada y el método de aprendizaje cooperativa "Jigsaw" puede promover capacidades y disposiciones de PC. Las conclusiones apuntan para el potencial de esta metodología en la adquisición de conocimientos, y el desarrollo de las capacidades de cuestionar, analizar, evaluar y sintetizar información así como de las disposiciones tales como la autoconfianza y la curiosidad.

Palabras clave: pensamiento crítico; Jigsaw y aprendizaje cooperativa; ingeniería; investigación guiada; enseñanza superior. 


\section{Introdução}

Face aos cenários e desafios do mundo atual, onde impera uma fast information que se espalha sem filtro, a passividade e a postura acrítica perante os fenómenos devem ser substituídas por um questionamento sistemático e um tratamento rigoroso dos assuntos. Como fazer crescer a capacidade individual para selecionar, avaliar a informação e transformá-la em conhecimento? Como interpretar a informação de modo a identificar e rejeitar postulados falsos, e a tomar decisões mais fundamentadas? Como ter consciência de que estamos a desenvolver um pensamento mais eficaz (Saíz, 2018) que nos leve a uma ação consciente e transformadora? Estas são algumas das questões que atravessam os debates atuais sobre Pensamento Crítico (PC) em todas as áreas científicas, não sendo a engenharia uma exceção.

Do consenso emergente sobre a importância do PC no Ensino Superior, resultante de várias iniciativas que destacam a necessidade de reformas educacionais para responder aos desafios sociais e económicos no século XXI (www.p21.org), estão a ser gradualmente incluídos nos currículos universitários, métodos pedagógicos mais ativos que potenciam o desenvolvimento do PC (Dominguez, 2018). Em geral apoiam-se na teoria construtivista da aprendizagem que afasta os estudantes de uma posição passiva, apoia a promoção de capacidades de PC dos estudantes como a análise, síntese, avaliação, decisão (Ennis, 2015), bem como os valores e atitudes que contribuem para a sua melhor integração no mercado laboral e para uma cidadania ativa.

Partindo do entendimento do PC como um pensamento de ordem superior ao qual se aplicam alguns standards intelectuais que permitem melhorar a sua qualidade, este artigo pretende contribuir para uma investigação empírica sobre estratégias ativas de ensino e aprendizagem que apoiam o desenvolvimento do PC, sendo ainda muito escassa e pouco sistematizada (Ennis, 2018). Apresenta e reflete sobre uma estratégia pedagógica que articula a Pesquisa Guiada (PG) com a Aprendizagem Cooperativa (AC) (mais especificamente o método Jigsaw) como uma abordagem inovadora para o desenvolvimento do PC (Figura 1) - abordagem essa que, do nosso conhecimento, não tem sido estudada. Tendo por base uma intervenção pedagógica semestral numa turma de alunos de primeiro ano do curso de licenciatura de engenharia mecânica, pretende-se mostrar como, utilizando esta estratégia, se pode atender às recomendações recorrentes de organismos internacionais de educação (como a Accreditation Board for Engineering and Technology (ABET)) que advogam por uma inclusão PC e de outras competências nos curricula de engenharia. 


\section{O Pensamento Crítico (PC)}

Embora não exista consenso sobre a sua definição por ser um conceito em evolução e alimentado a partir de várias perspetivas (filosofia, psicologia, educação), o PC, de forma esquemática, apresenta duas grandes dimensões, uma individual e outra sociocultural. A primeira, preocupada com as atividades intelectuais específicas, permite elevar a qualidade do pensar para chegar a julgamentos ou decisões mais fundamentadas. O PC é, nesta dimensão, um pensamento "razoável e reflexivo focado em decidir no que acreditar ou o que fazer" (Ennis, 2015, p. 1), "a arte de refletir sobre o pensamento de uma forma intelectualmente disciplinada" (Paul, 2005, p.28), 'o exame cuidadoso de uma questão para chegar a um julgamento fundamentado" (Bailin \& Battersby, 2016, p. 6). As capacidades em causa são as de saber analisar, explicar, interpretar, fazer inferências, avaliar, decidir em que acreditar, resolver problemas de forma eficaz, questionar de forma profunda, ser capaz de detetar fragilidades e aplicar rigor ao seu modo de pensar (autorregulação), entre outras (Paul \& Elder, 2008). Ainda nesta dimensão, uma grande parte de autores adiciona uma sub-dimensão atitudinal preocupada em cultivar disposições como a capacidade de suspender o julgamento antes de formular uma opinião, a abertura, a tolerância, a curiosidade, o respeito pelas diferenças, a busca do rigor, a escuta empática, a coragem de se questionar e questionar o mundo, entre outras (Facione, Sanchez, Facione, \& Gainen, 1995). É geral o entendimento de que o desenvolvimento das capacidades de PC é indissociável do cultivar das disposições (Facione et al., 1995).

A segunda dimensão do PC, a sociocultural, surge, mais recentemente, por autores e simpatizantes do movimento da pedagogia crítica. Para estes o termo 'criticalidade' ultrapassa o de PC na medida em que não só incorpora a argumentação, reflexão e o julgamento (as capacidades) e o ser (disposições) mas também a ação, projetando o indivíduo como elemento transformador da sociedade em que vive (Davies \& Barnett, 2015). Nesta dimensão, o ensino superior tem um papel crucial na transformação dos indivíduos e da sociedade. Deve preparar os estudantes não só para adquirir e consolidar capacidades de pensamento de ordem superior, mas também incitá-los a posicionarem-se no mundo que os rodeia desenvolvendo uma identidade crítica que abrange a sua participação como cidadãos e profissionais. Na área da engenharia os estudantes devem, não só adquirir competências técnicas específicas da profissão, como também refletir sobre o impacto das suas decisões na sustentabilidade económica, social e ambiental. O desafio dos docentes passa a ser o de como transformar os estudantes em sujeitos ativos de pensamento e de ação, o que pressupõe uma aprendizagem que envolva um questionamento e relação mais próxima com a realidade.

Considerando estas dimensões do PC, a ponte com as estratégias ativas de aprendizagem parece realizar-se através de práticas que desenvolvam nos estudantes, entre 
outras, a curiosidade, a capacidade de questionar evidências, raciocínios e atitudes, a procura e discussão de soluções, a partilha de opiniões, por oposição à prática de memorização do conhecimento de uma disciplina.

\section{Pesquisa guiada (PG) e Pensamento Crítico (PC)}

A Pesquisa guiada (PG) é uma abordagem pedagógica ativa, centrada na aprendizagem do estudante capaz de desenvolver o PC. Recorre a estratégias como o questionamento, a análise, a avaliação de fontes (Todd, 2017), que encorajam uma prática reflexiva e exploratória munindo os alunos de ferramentas e recursos capazes de estimular o desenvolvimento de uma aprendizagem sistémica.

Desenvolvida a partir das necessidades sentidas e desafios lançados por um contexto expansivo de informação heteróclita complexa, de conectividades facilitadas pelas tecnologias da informação, e por um decréscimo de interesse dos estudantes pelas áreas das ciências (Kang \& Keinonen, 2018), a PG tem vindo, nas últimas décadas, a afirmar-se como um método pedagógico interativo, científico e cooperativo diferenciado que capacita os estudantes a destrinçarem uma miríade de informações contraditórias (Grafstein, 2017), a selecionarem as verdadeiras fontes, a aprofundarem, compreenderem e a argumentarem sobre questões relevantes através de intervenções orientadas e supervisionadas (Kuhlthau, 2010) e a construírem o seu próprio percurso gnosiológico (Kang \& Keinonen, 2018). Em 17 estudos que foram conduzidos entre os anos 2000 a 2012 para avaliar os impactos da aplicação da PG na aprendizagem dos estudantes nas suas diferentes dimensões, afetiva, cognitiva e atitudinal, verificaram-se efeitos positivos nas práticas epistémicas ligadas às ciências em geral e às engenharias em especial (van Loon \& Lai, 2014), realçando as componentes motivacionais e comportamentais como motores geradores de curiosidade e de conhecimento. Para estes autores, o foco está em criar estratégias pedagógicas que auxiliem os estudantes a desenvolver capacidades e disposições que lhes permitam refletir na tomada de decisões e resolver problemas complexos e ambíguos da sua área específica (van Loon \& Lai, 2014). O ciclo de aprendizagem processa-se através de evidências e de práticas construtivistas sustentadas por um questionamento significativo, e de modo a tornar os aprendentes construtores do conhecimento (Kang \& Keinonen, 2018).

Esta estratégia não se dissocia dos currículos a ministrar nos diferentes graus de ensino, exigindo à Equipa de Instrução (EI), composta por professores e bibliotecários, um desenho articulado do contexto, do planeamento de tarefas e ações, de partilhas na recolha, organização e progressão das atividades e, ainda, de supervisão. O professor e o bibliotecário definem os objetivos de uma unidade curricular (UC), selecionam o tema a explorar e organizam os recursos a dispor para cada sessão. Este é um trabalho colaborativo entre a El, vista como facilitadora das aprendizagens (Leonard \& 
Nwanekezi, 2018). O registo das evoluções e os níveis de proximidade, de inclusão, de interação e de acompanhamento que consolidam as dimensões afetiva, cognitiva e comportamental (Todd, 2017), permitem afinar e relevar os procedimentos reflexivos e inquiridores que capacitam uma maior autonomia (Kang \& Keinonen, 2018), confiabilidade, diálogo e interação.

O modelo, segundo Kuhlthau \& Todd (2009), estrutura-se em sete dimensões articuláveis e não exclusivas, designadas por processos de iniciação, seleção, exploração, formulação, recolha, apresentação e avaliação. A estrutura flexível estabelecida, baseada num trabalho individual e cooperativo, é sustentada por um método de questionamento aberto e procura encorajar o estudante a mergulhar em diversos procedimentos, ações e informações recorrendo a diferentes fontes e suportes para consolidar a sua aprendizagem, onde a presença e a ação do facilitador-instrutor se torna fundamental (Leonard \& Nwanekezi, 2018).

\section{JIGSAW e Pensamento crítico}

O Jigsaw, ou método dos puzzles, é um método de Aprendizagem cooperativa (AC) que requer a divisão das tarefas de aprendizagem com o objetivo de estimular a cooperação entre os alunos. Criado por Aronson, Blaney, Stephan, Sikes e Snapp (1978), alguns autores sublinham as suas vantagens para o estudo de conceitos (Kilic, 2008; Tran \& Lewis, 2012) e desenvolvimento do PC (Ghaith \& El-Malak, 2004) em qualquer área do conhecimento. As evidências empíricas do impacto positivo na melhoria das aprendizagens dos alunos conduziram à sua inclusão no grupo dos oito métodos de AC mais eficazes (Johnson, Johnson \& Stanne, 2000).

Com uma sintaxe própria, a utilização do Jigsaw exige, num primeiro momento, a formação de grupos cooperativos de 3 a 4 elementos (grupos de base), heterogéneos, com distribuição e rotatividade de papéis. Estes grupos devem ser estruturados para que sejam garantidas a interdependência positiva, a responsabilidade individual e de grupo, a interação estimuladora, o desenvolvimento de competências sociais e o processo de avaliação do grupo. O professor estabelece os objetivos de aprendizagem, escolhe o tema a estudar e prepara os recursos que vão ser utilizados.

A aplicação do método inicia-se com a divisão do tema a estudar em tantas partes quanto o número de elementos dos grupos. Se há 4 alunos por grupo, o tema deve ser dividido em 4 partes. Cada elemento do grupo de base recebe e estuda uma parte do tema em análise. Quando termina esta tarefa (o tempo e os recursos podem ser diversos dependendo dos objetivos de aprendizagem) juntam-se, em grupos de peritos, todos os elementos que têm em comum a mesma parte do tema em estudo. Para facilitar a organização dos passos a seguir sugere-se o uso de folhas de cores diferentes para cada parte do tema/matéria a estudar/investigar. Cada grupo de peritos 
fica responsável pelo estudo aprofundado das matérias e desenha a melhor forma de a ensinar aos colegas quando os seus elementos regressarem ao seu grupo de base. A reestruturação cognitiva que acontece quando os alunos têm que explicar aos seus colegas o que já sabem contribui para a melhoria das aprendizagens.

É um método de AC relevante para o estudo na área das engenharias (Felder, Woods, Stice, \& Rugarcia, 2000) por permitir a resolução de problemas que envolvam grandes quantidades de informação (Ghaith \& El-Malak, 2004), promover o trabalho em equipa, a aprendizagem de conceitos e o desenvolvimento do PC. Contudo, são ainda escassos os estudos sobre os efeitos da combinação entre a PG e o Jigsaw, como método cooperativo, e o desenvolvimento das capacidades e disposições de PC.

\section{Metodologia de investigação}

Por serem escassos os estudos que combinam a PG, a AC (Jigsaw) e o PC, a presente investigação de natureza qualitativa pretende contribuir para a reflexão sobre a temática, apoiando o desenvolvimento de experiências futuras por parte de docentes do ensino superior. Assim, através de um estudo de caso exploratório, descreve-se o contexto e as diferentes etapas da abordagem pedagógica implementada. Também são apresentadas algumas reflexões em torno dos resultados obtidos, quer pela observação direta e participante em sala de aula efetuada pela El, quer pelo desempenho académico (e.g., mapas concetuais, análise FRISCO, teste final, apresentações orais) e perceções dos alunos recolhidas através de um inquérito final sobre o desenvolvimento de toda a atividade.

\section{Desenho da experiência}

Trata-se de uma intervenção pedagógica na qual, ao longo de um semestre letivo, que correspondeu a 15 sessões de 120 minutos cada, procurou-se incentivar os estudantes a adquirir conhecimentos, descobrir, explorar e desenvolver as capacidades e disposições do PC a partir da formulação de uma questão central sobre um tema da UC (a responder até ao fim do semestre). Esta serviu de base ao desenvolvimento de todas as atividades de aprendizagem realizadas, culminando na partilha e apresentação dos resultados. O objetivo era preparar os estudantes para pensar e usar informação de modo crítico e gerarem novos conhecimentos (i.e. gestão de resíduos industriais: tipos, origem, natureza, aplicações, impactos) a partir de questões de natureza cognitiva, gradativamente mais complexas e desenvolver também atitudes mais proativas e conectivas (ver disposições de PC, Figura 1).

Usou-se a estratégia de PG combinada com o Jigsaw para promover as capacidades de PC, especialmente as de questionar (i.e. formular perguntas descritivas, analíticas e avaliativas), analisar (examinar e decompor as partes), sintetizar (relevar o 
essencial) e avaliar (valorizar), e as disposições como curiosidade, confiança, autonomia, imparcialidade, Humildade Intelectual (Facione, 1995).

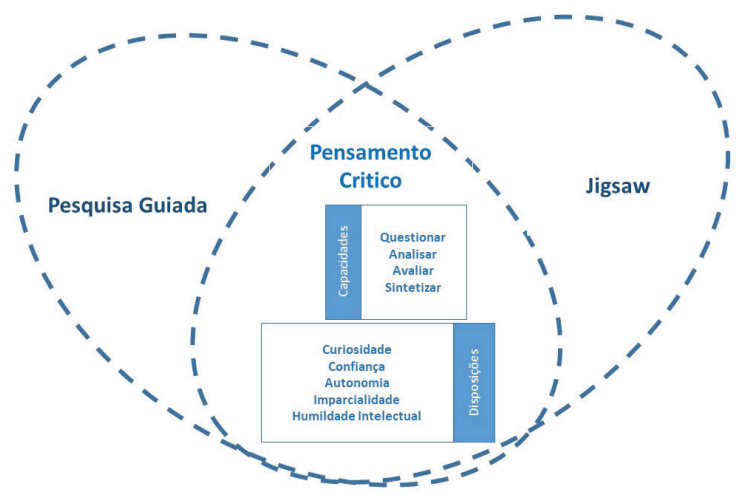

Figura 1 - Modelo conceptual de intervenção

\section{Participantes}

Participaram neste estudo 30 estudantes de uma universidade pública do Norte de Portugal a frequentar a unidade curricular de gestão industrial, correspondente ao $1 .{ }^{\circ}$ ano da licenciatura em engenharia mecânica, com idades compreendidas entre 18 a 20 , sendo $20 \%$ de sexo feminino.

\section{Equipa de Instrução (EI)}

Para o planeamento e supervisionamento das sessões e seleção temática a investigar, envolveu-se uma El constituída pela docente da UC e a bibliotecária da instituição. As questões de natureza curricular estiveram a cargo da docente da UC, as de PG da bibliotecária. Em conjunto orientaram e supervisionaram as atividades de descoberta, exploração, questionamento e avaliação. A função facilitadora e de acompanhamento da El procurou que, durante as etapas de assimilação e aprofundamento das questões, nomeadamente nos processos de identificação, seleção, uso e avaliação da informação, do questionamento, da análise, da compreensão e reflexão temáticas (Anexo 1), a aprendizagem se tornasse mais significativa e duradoura e que a dimensão crítica emergisse como resultado de debate e discussão de ideias do grupo.

\section{Procedimentos utilizados para a aprendizagem}

De acordo com as orientações para a AC (Johnson, Johnson, \& Stanne, 2000) a docente constituiu cinco grupos heterogéneos de seis elementos. O tema comum 
aos grupos (resíduos industriais) foi dividido, nos grupos, em tantas partes quanto os elementos do grupo: resíduos nos setores têxtil, aerospacial, madeiras, automóvel, construção civil e metalurgia.

Para cada grupo foi indicado um líder com a missão de, por um lado, mobilizar e motivar os restantes elementos e, por outro, assegurar a concretização do desafio proposto e desenvolver as competências sociais no grupo. Outros papéis foram distribuídos entre os elementos (porta-voz, secretário, controlador do tempo).

As fases da PG enunciadas por Kuhlthau \& Todd (2009) foram introduzidas de forma encadeada e flexível, articuladas com o método cooperativo Jigsaw (Aronson et al, 1978) conforme a Figura 2.

\section{Pesquisa Guiada e Jigsaw: fases e recursos}

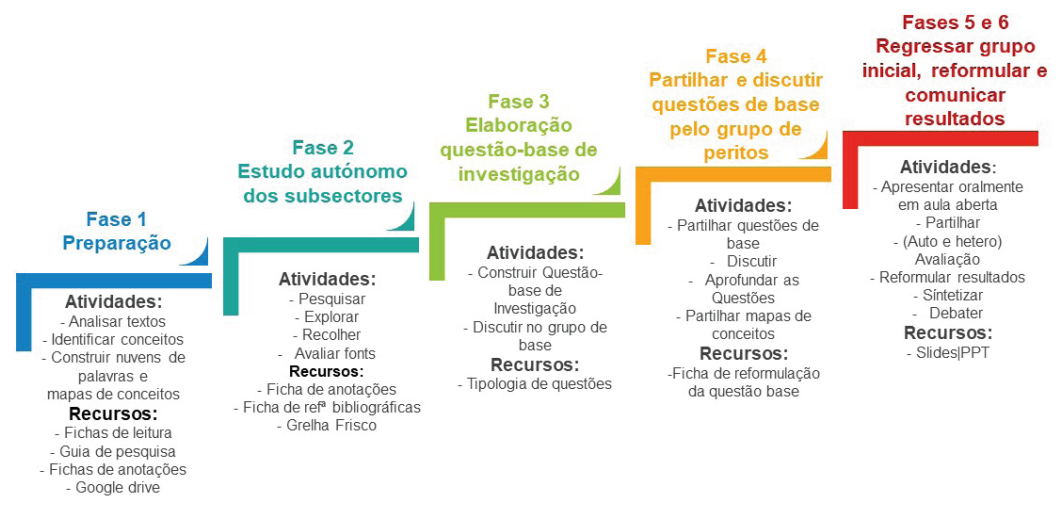

Figura 2 - Fases da estratégia, atividades e recursos pedagógicos de apoio

\section{1. ${ }^{a}$ fase: Preparação (3 sessões)}

Para adquirirem experiência de trabalho cooperativo e de técnicas de pesquisa de informação, distribuiu-se, a todos os estudantes, organizados em grupos de base, um texto comum (artigo de opinião sobre o impacto ambiental dos smartphones). Pretendia-se que os estudantes aprendessem a avaliar a fonte, identificassem os conceitos fundamentais, com a representação gráfica em nuvens de palavras e mapa concetual, e identificassem as questões explícitas e implícitas do texto (anexo 1). Utilizou-se a técnica cooperativa "think-pair-share" (pensar - formar pares -partilhar) (Lyman, 1987) para construir uma ficha de trabalho comum.

O supervisionamento consistiu em sessões práticas, ministradas pela bibliotecária, sobre o uso e a credibilidade de fontes informacionais, nuvens de palavras e mapas concetuais (Figura 3). Utilizaram-se os seguintes recursos: i) ficha de anotações, ii) guia de 
pesquisa iii) ficha de avaliação das fontes, iv) ficha de leitura, v) ficha de construção de mapas conceptuais (e a sua respetiva avaliação) e vi) nuvens de palavras. Esta fase consistiu também em familiarizar os alunos com o uso do Google Drive para organizarem, por grupos e por estudante, todo o percurso de investigação e os recursos utilizados e a utilizar.

\section{2. ${ }^{a}$ fase: Estudo autónomo dos subsectores (2 sessões e trabalho fora da sala de aula)}

Cada estudante iniciou o seu percurso de pesquisa sobre os resíduos industriais no setor industrial que Ihe foi atribuído no seu grupo, selecionou artigos, avaliou a credibilidade das fontes (Kuhlthau, Maniotes, \& Caspari, 2012) através do registo das ideias principais dos textos representadas num mapa conceptual (Figura 3).

O acompanhamento consistiu, por um lado, numa instrução-orientação sobre técnicas de pesquisa avançadas para recuperação eficaz e de qualidade da informação, o seu uso ético, a elaboração de referências bibliográficas, a seleção de palavras-chave e o registo das fontes segundo as normas em vigor, com recurso a ferramentas de gestão bibliográfica (ficha de citações e de referências bibliográficas) e, por outro lado, no feedback sobre a evolução dos mapas de conceitos.

O propósito era capacitar os estudantes a orientarem-se autonomamente na pesquisa mais refinada, analisarem, selecionarem e sintetizarem a informação recolhida com autoconfiança, para construírem (na fase seguinte) a questão-base de investigação do grupo e, a partir dela, desenvolverem as restantes atividades da investigação. Pretendia-se também que se exercitassem de forma sistemática a usar capacidades de PC exigindo-Ihes que a análise textual fosse efetuada com base na grelha FRISCO (Foco, Razões, Inferências, Clareza, Overview), acrónimo de um guia de análise criado por Ennis (1996) que ajuda a treinar as capacidades de PC.

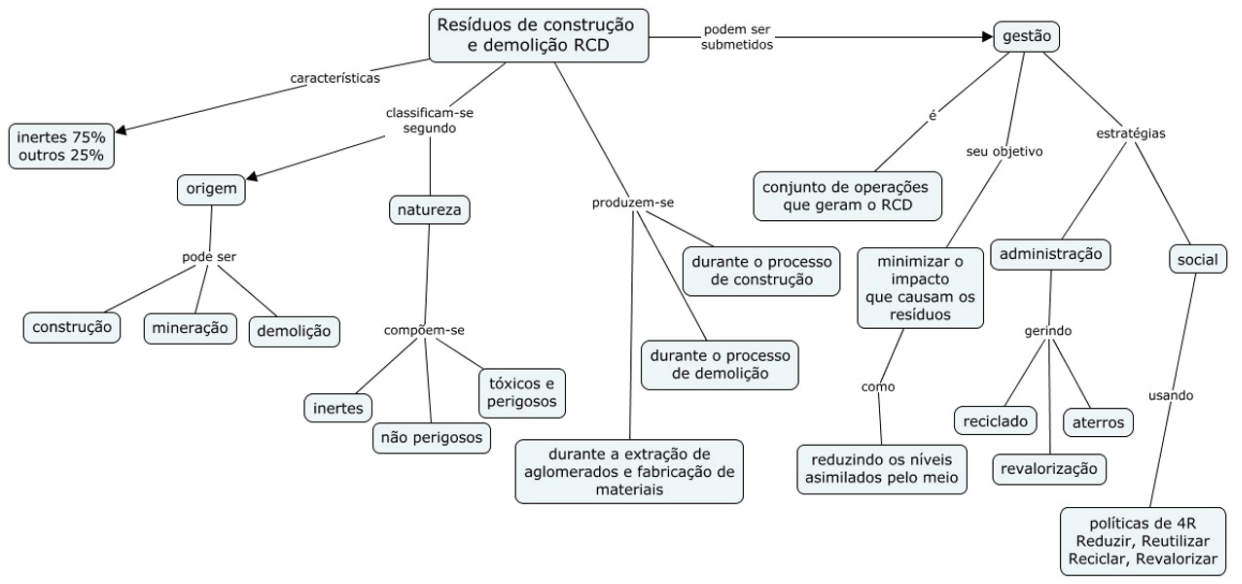

Figura 3 - Exemplo de um mapa conceptual de um aluno 


\section{3. ${ }^{a}$ fase: Elaboração da questão-base de investigação (2 sessões)}

Os alunos, em grupo, confrontaram os mapas conceptuais elaborados individualmente, (por setores industriais) para elaborarem uma questão única de pesquisa, fundamentada e alimentada pelas diferentes perspetivas setoriais.

Exemplos: "Como será possível reutilizar o lixo resultante da atividade industrial, tanto dentro do próprio setor como em outros?"

"A nível político e económico, o que impede a possível diminuição do impacto ambiental nos setores industriais existentes?"

"Qual é o impacto positivo da reciclagem?"

A atividade foi reforçada pela El com a apresentação de exercícios sobre a natureza e tipologia de questões (descritivas, analíticas e de avaliação) para aprofundar os níveis de compreensão temática (Hilsdon \& Bitzer, 2007).

\section{4. ${ }^{a}$ fase: Partilha e discussão das questões de base pelos grupos de peritos e aprofundamento do conhecimento (3 sessões)}

Elaborada a questão inicial, os grupos de base desagregaram-se e constituíram-se os grupos de peritos juntando todos os elementos de cada setor industrial como se apresenta na Figura 4.
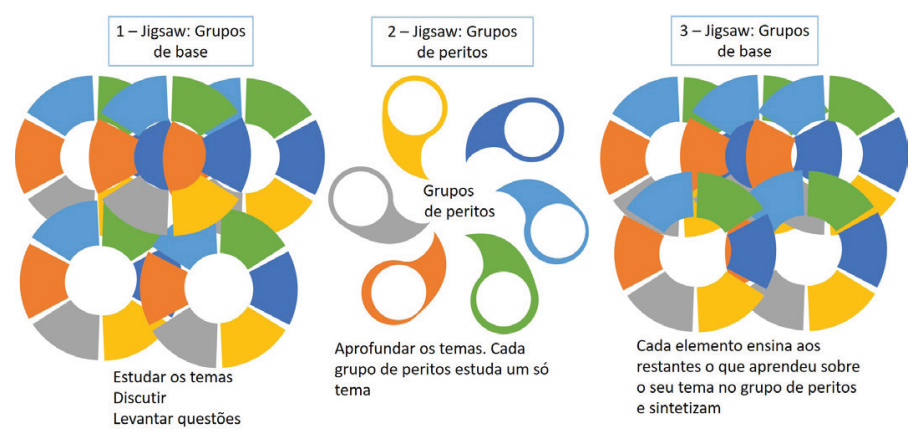

Figura 4 - Fases do Jigsaw

Nesta etapa, em grupo de peritos, os alunos aprofundaram em conjunto o seu setor. Rotativamente, cada elemento expunha oralmente a pergunta do seu grupo de base e o seu mapa concetual. Os restantes elementos registavam, numa ficha de reformulação de questões (Anexo 2) o seu entendimento sobre a questão exposta pelo colega. Escreviam e colocavam questões ao colega apresentador que permitiu esclarecer aspetos como: se a questão era específica ou geral, se estava devidamente fundamentada, se podia ser reformulada. Nesta etapa, todos os elementos contribuíram para o enriquecimento da questão a partir do aprofundamento efetuado sobre o setor que estudaram previamente. Depois, cada aluno escreveu na sua ficha de trabalho 
individual uma resposta à questão colocada pelo apresentador e partilhou-a com os restantes elementos. Após a discussão de todas as respostas individuais, o grupo devia chegar a uma resposta consensual. Este exercício permitiu reestruturar cooperativa e cognitivamente os conhecimentos, estimular uma atitude aberta e desenvolver competências sociais (saber ouvir, expor as suas ideias, respeitar os turnos, colaborar, incluir as ideias, etc.).

\section{5. ${ }^{a}$ fase: Regresso aos grupos iniciais para (re)formulação da questão inicial e respetiva resposta (3 sessões)}

Os peritos regressaram ao seu grupo de base, enriquecidos com os contributos recolhidos na etapa anterior, partilharam com os elementos do grupo de base a resposta elaborada com o grupo de peritos, e submeteram-na à avaliação do seu grupo. Elaboraram uma síntese a partir das diferentes perspetivas setoriais, reformularam a questão inicial e fundamentaram a resposta. Nesta etapa, o esforço focou-se na capacidade de síntese.

\section{6. ${ }^{a}$ fase: Comunicação dos resultados (2 sessões e trabalho fora de aula)}

O projeto experimental terminou com cada grupo a preparar e expor, oralmente, o trabalho final, seguindo-se um debate sobre as conclusões, entre os grupos presentes. Foram colocadas questões de esclarecimento sobre alguns pontos, apresentadas pelos diferentes colegas e pela El a cada grupo expositor. Terminado este ciclo, distribuiu-se um inquérito de aferição das perceções dos estudantes em relação à estratégia utilizada. Tendo disponibilizado antecipadamente (no início) os critérios de avaliação e os coeficientes de ponderação para cada atividade (Tabela 1)., a El avaliou também todo o processo individual e de grupo dando contínuo feedback. O teste final versou sobre saber citar e referenciar e avaliar informação. A classificação final foi positiva.

Tabela 1 - Avaliação das atividades

\begin{tabular}{|c|c|c|c|c|c|}
\hline & $\begin{array}{c}\text { Mapas } \\
\text { conceptuais }\end{array}$ & $\begin{array}{c}\text { Análise } \\
\text { FRISCO }\end{array}$ & $\begin{array}{c}\text { Contributo individual } \\
\text { em grupo de peritos }\end{array}$ & $\begin{array}{c}\text { Apresentação } \\
\text { oral }\end{array}$ & $\begin{array}{c}\text { Teste } \\
\text { Final }\end{array}$ \\
\hline $\begin{array}{c}\text { Peso na nota } \\
\text { final }\end{array}$ & $25 \%$ & $25 \%$ & $20 \%$ & $25 \%$ & $5 \%$ \\
\hline $\begin{array}{c}\text { Média de } \\
\text { Classificação }\end{array}$ & 15,6 & 14,2 & 13,8 & 16,8 & 15,7 \\
\hline $\begin{array}{c}\text { Nota mínima } \\
\text { da turma }\end{array}$ & 13 & 12 & 9 & 14 & 14 \\
\hline $\begin{array}{c}\text { Nota máxima } \\
\text { da turma }\end{array}$ & 17 & 18 & 17 & 18 & 18 \\
\hline
\end{tabular}




\section{Reflexões sobre as dimensões trabalhadas e considerações finais}

O que podemos retirar como conclusões/reflexões sobre esta abordagem conjunta de PG e Jigsaw no desenvolvimento de conhecimentos, capacidades e disposições de PC dos alunos? Considerando os dados objetivos disponíveis da avaliação (limitados às notas) e as observações registadas, entendemos que 4 dimensões poderão ter sido desenvolvidas (Figura 5).

\section{Dimensões trabalhadas}
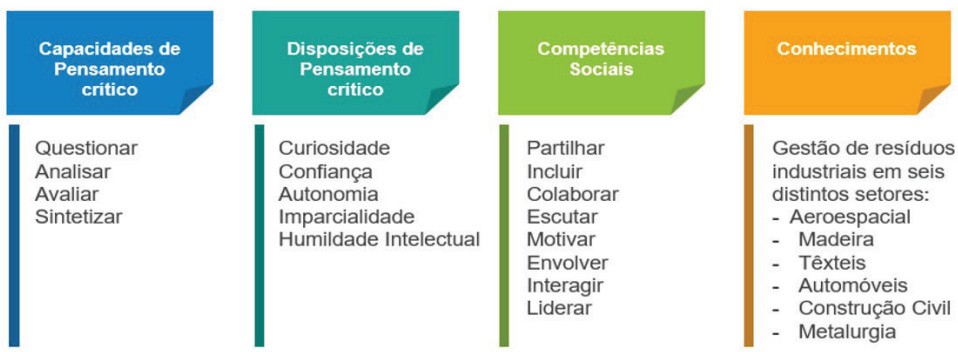

Figura 5 - Resultados expectáveis da metodologia utilizada

Em relação aos conhecimentos, ficou patente, pelos mapas conceptuais, qualidade dos trabalhos finais e das apresentações orais que os alunos adquiriram os conceitos fundamentais da UC estabelecidos nos objetivos iniciais. Não só conseguiram "especializar-se" e aplicar os conceitos de gestão de resíduos num sector específico, e nos dos seus colegas, como também construíram uma visão mais abrangente e enriquecedora sobre a temática, guiando-se pelas perguntas de investigação num percurso individual e coletivo. As apresentações orais constituíram um momento de grande dinamização, motivação e participação. As notas finais desse item refletem esse resultado (Tabela 1) que incluíram a avaliação da questão-base, a sua fundamentação, soluções apresentadas, presença de fontes credíveis citadas e clareza oral.

Verificou-se uma evolução geral nas capacidades de análise, interpretação, síntese e avaliação da informação refletida na maior parte dos mapas conceptuais que foram sendo melhorados e aprofundados ao nível das relações conceptuais hierárquico-associativas e do uso da grelha FRISCO (Ennis, 1996). A abordagem cooperativa com o Jigsaw contribuiu para melhorar o nível de questionamento dos alunos (e de capacidade de resposta às questões), refletido na melhoria das questões dos grupos-base após debate nos grupos de peritos e no registo das fichas individuais de reformulação das questões. As suas capacidades de síntese foram exploradas graças à dinâmica "grupo base-peritos-grupo base". 
Relativamente às disposições, a El observou e registou, num diário semanal, um aumento de atenção e envolvimento (motivação), uma aprendizagem organizada e gradual de partilha de ideias, uma maior desinibição de alunos mais tímidos (autoconfiança), e em geral uma maior curiosidade (alguns grupos e/ou alunos pesquisaram, registaram e organizaram na Google drive mais artigos dos que os exigidos).

Considerando que pode ser útil para este artigo, refletimos aqui sobre alguns resultados que decorreram de um inquérito final com duas perguntas abertas, distribuído aos 30 alunos na última sessão em aula. As respostas foram sujeitas a uma análise qualitativa da qual emergiram categorias tendo-se realizado uma contagem do número de vezes que as mesmas foram referenciadas. As perguntas foram: 1) o que, na opinião deles deveria ser mantido e porquê; 2) o que, na opinião deles deveria ser mudado e porquê.

Os principais resultados do inquérito revelaram que 1) $70 \%$ apreciaram e manteriam o trabalho de grupo; 30\% gostaram da experiência na sua totalidade (resposta: gostei de tudo); 20\% apreciou a técnica do Jigsaw. As razões expostas revelam que o debate suscitado pelas perguntas e as dinâmicas do Jigsaw foram sentidas como benéficas "ao contrário do que acontece em outras disciplinas em que essa dinâmica não existe"; 2) 26\% dos alunos teriam gostado de trabalhar sobre outro tema (à escolha) e $37 \%$ realçaram a necessidade de reduzir o volume de trabalho.

No que se refere à El, esta teve um papel fundamental na planificação e realização de todo o percurso (Leonard \& Nwanekezi, 2018), que foi exigente (por ser novo). Procurou gerar novos contextos de aprendizagem, preparar e disponibilizar recursos de suporte, acompanhar a progressão e o registo das evoluções, definir claramente os objetivos de aprendizagem para cada sessão, delinear etapas, suscitar motivação e interesse e avaliar (feedback in loco e na Google Drive). Procurou provocar uma atitude mais crítica e construtiva. Através da instrução de estratégias de pesquisa, de questionamento, e de uso de outras ferramentas, procurou desenvolver nos alunos uma compreensão mais integrada do tema.

A complementaridade dos papéis da bibliotecária com a docente responsável pela UC permitiu que cada uma se concentrasse no seu domínio e, ao mesmo tempo, beneficiasse do acompanhamento da outra. O diário elaborado após cada sessão foi uma importante ferramenta, não só de construção da memória do percurso de aprendizagem (para futuras edições), mas também de autorreflexão para ajustamento e aperfeiçoamento das atividades e práticas pedagógicas implementadas. Em futuras edições, será importante que os alunos tenham mais oportunidades e sejam avaliados mais regularmente na prática de citação e referenciação, aspetos um pouco mais técnicos relativos à $P G$ e onde houve mais dificuldades. Um feedback ainda mais imediato e regular poderá reforçar a aprendizagem. 
Como reflexão final, espera-se que a descrição desta experiência possa ser útil a docentes que queiram aplicar uma metodologia ativa num percurso de investigação cooperativa que promova o PC (Jigsaw). Corroborando alguns autores como Todd (2017), a PG evidenciou sustentar esta experiência com uma aprendizagem significativa. Combinada com a dinâmica do Jigsaw, especialmente no movimento de vai e vem dos participantes entre o grupo de base e o grupo de peritos, permitiu um aprofundamento das questões e proporcionou boas oportunidades de desenvolvimento de capacidades de análise e síntese. Foi possível os alunos destrinçarem uma miríade de informações, por vezes contraditórias e de várias fontes, compreenderem e argumentarem sobre questões relevantes assim como adquirirem e aplicarem novos conceitos (Tran \& Lewis, 2012), ao mesmo tempo que iam construindo o seu próprio percurso gnosiológico (Kang \& Keinonen, 2018), consolidando a sua motivação e autonomia.

Como reflexão final, esta abordagem pedagógica parece-nos ter sido adequada para o curso de engenharia. Ela confere um potencial importante na aquisição de competências individuais e sociais úteis para a aprendizagem ao longo da vida (Naimpally, Ramachandran \& Smith, 2012). Para trabalhos futuros será importante consolidar esta hipótese, realizando um pré e pós-teste do PC de modo a avaliar de forma mais consistente o contributo desta UC no desenvolvimento das competências desejadas e delinear uma investigação de natureza experimental com 3 grupos de alunos, um apenas com o Jigsaw, outro só com a PG e outro com ambas de modo a medir o impacto desta estratégia. Poderá alargar-se à investigação a outras áreas do saber e profissionais.

\section{Agradecimentos}

Este trabalho insere-se no Projeto 'Pensamento Crítico nos Currículos do Ensino Superior Europeu - CRITHINKEDU', com o número de referência 2016-1-PT01-KA203-022808, financiado pela Comissão Europeia no âmbito do Programa Erasmus+.

\section{Referências Bibliográficas}

Aronson, E., Blaney, N., Stephan, C., Sikes, J., \& Snapp, M. (1978). The Jigsaw Classroom. Beverly Hills, CA: Sage Publications.

Bailin, S. \& Battersby, M. (2016). Reason in the balance: an inquiry approach to critical thinking. Indianopolis: Hackett Publishing.

Davies, M. \& Barnett, R. (Eds.). (2015). The Palgrave handbook of critical thinking in higher education. New York: Palgrave Macmillan.

Dominguez, C. (coord.) (2018). A European review on Critical Thinking educational practices in Higher Education Institutions. Vila Real: UTAD.

Ennis R. H. (1996). Critical Thinking. Upper Saddle River, NJ: Prentice Hall. 
Ennis, R. H. (2015). Critical thinking: A streamlined conception. In M. Davies\& R. Barnett (Eds). The Palgrave handbook of critical thinking in higher education (pp. 31-47). New York: Palgrave Macmillan.

Ennis, R.H (2018). Critical thinking across the curriculum: a vision. Topoi, 37(1), 165-184. [Disponível em https: //doi.org/10.1007/s11245-016-9401-4, consultado em 18 de Novembro 2018].

Facione, P. A., Sanchez, C. A., Facione, N. C., \& Gainen, J. (1995). The disposition toward critical thinking. The Journal of General Education, 44(1), 1-25.

Felder, R. M., Woods, D. R., Stice, J. E., \& Rugarcia, A. (2000). The future of engineering education II. Teaching methods that work. Chemical Engineering Education, 34(1), 26-39.

Ghaith, G., \& El-Malak, M. A. (2004). Effect of Jigsaw II on literal and higher order EFL reading comprehension. Educational Research and Evaluation, 10(2), 105-115.

Grafstein, A. (2017) - Information literacy and critical thinking: context and practice. In D. Sales \& M. Pinto (Eds.). Pathways into information literacy and communities of practice: Teaching approaches and case studies (pp. 3-28). Amsterdam: Chandos Publishing.

Hilsdon, J. \& Bitzer, E. M. (2007). To become an asker of questions. A 'functional-narrative' model to assist students in preparing postgraduate research proposals. South African Journal of Higher Education, 21(48), 1194-1206.

Johnson, D. W., Johnson, R. T., \& Stanne, M. E. (2000). Cooperative learning methods: a metaanalysis. Minneapolis: University of Minnesota Press.[Disponivel em https://www.researchgate.net/ publication/220040324_Cooperative_learning_methods_A_meta-analysis, consultado em 24 /10/ 2018].

Kang, J. \& Keinonen, T. (2018). The effect of student-centered approaches on students' interest and achievement in science: Relevant topic-based, open and guided inquiry-based, and discussionbased approaches. Research in Science Education, 48(4), 865-885.

Kilic, D. (2008). The effect of the Jigsaw technique on learning the concepts of the principles and methods of teaching. World Applied Sciences Journal, 4(1), 109-114.

Kuhlthau, C. \& Todd, R. (2009). Indagación guiada: un Sistema para el aprendizaje en las instituciones educativas del siglo XXI, mediante el uso de bibliotecas escolares. [Disponível em: http://eduteka. icesi.edu.co/articulos/indagacion-guiada-fundamentos, consultado em 12/11/2018].

Kuhlthau, C. (2010). Guided inquiry: School libraries in the 21st century. School Libraries Worldwide, 16(1), 17-28. [Disponível em http://citeseerx.ist.psu.edu/viewdoc/summary?doi=10.1.1.721.8981, consultado a 24/09/2018].

Kuhlthau, C. C., Maniotes, L. K., \& Caspari, A. K. (2012). Guided Inquiry Design: a Framework for Inquiry in Your School. Santa Barbara, CA: Libraries Unlimited.

Leonard, N. \& Nwanekezi, A. (2018). Effects of guided inquiry and task hierarchy analysis model in cooperative learning strategy on chemistry students' performance in Imo State. European Scientific Journal, 14(25), 54-62. [Disponível em http://eujournal.org/index.php/esj/article/view/11235, consultado a 1/10/2018].

Lyman, F (1987). Think-pair-share: an ending technique. MAA-CIE Cooperative News, 1, 1-2. 
Naimpally, A.,Ramachandran, H. \& Smith, C. (2012). Lifelong Learning for Engineers and Scientists in the Information Age. Amesterdan: Elsevier.

Paul, R. (2005). The State of Critical Thinking Today. New Directions for Community Colleges, 130, 27-38. [Disponível em https://doi.org/10.1002/cc.193, consultado em 10/09/2018].

Paul, R. \& Elder, L. (2008). Critical Thinking: Strategies for Improving Student Learning, Part II. Journal of Developmental Education, 32(2), 34-35.

Saíz, C. S. (2018). Pensamiento crítico y eficacia. Madrid: Ediciones Pirámide.

Todd, R. (2017). Information Literacy: agendas for a sustainable future. Journal of Information Literacy, 11(1), 120-136. [Disponível em https://ojs.lboro.ac.uk/JIL /article/view/PRA-V11-11-7, consultado a 14/10/2018].

Tran, V. D. \& Lewis, R. (2012). The effects of Jigsaw learning on students' attitudes in a Vietnamese higher education classroom. International Journal of Higher Education, 1(2), 120-136. [Disponível em http://www.sciedu.ca/journal/index.php/ijhe/article/view/1115/613, consultado em 14/10/2018].

Van Loon, J. \& Lai, H. (2014). Information Literacy Skills as a Critical Thinking Framework in the Undergraduate Engineering Curriculum. Library Scholarly Publications, Paper 80. [Disponível em http://digitalcommons.wayne.edu/libsp/80, consultado em 19/12/2018]. 


\section{Anexo 1 - Ficha de trabalho no grupo dos peritos}

\section{FICHA DE TRABALHO DIA 28 de MAIO- LIXO INDUSTRIAL}

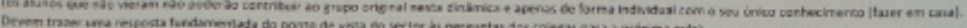
Nome numero

1. Os alunos de cada sector industrial reúnem-se no grupo de "experts"

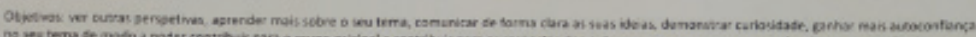

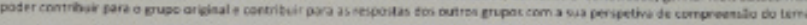
2. Tarefas:

a) 1 aluno expõe claramente como compreendeu o seu tema atraves do seu mapa conceptual e apresenta a pergunta le a sua fundamentaçðo / do seu grupo original. No decorrer da actividade este aluno deve registar, por escrito, tudo o que the for comunicado numa folha de papel.

Nome do aluno que expōe:

b) Escreva por suas próprias palavras o que entendeu que era a pergunta $e$ a fundamentaçăo, a que tipo de resposta se quer chegar.

c) Escreva a seguir as questôes que the permita avaliar a pergunta de acordo com a compreensdo apresentada pelo colega e com a sua própria compreensăo do tema: clareza, pertinência, releváncia, elementos năo considerados na fundamentaç̆o, coerẻncia em relação à compreensăo do tema apresentado, se é especifica, se égeral.

d) E partilhado e discutido em grupo de pares primeiro e depois para o grupo tudo o que tiverem anotado.

e) Depois da discussăo no grupo, se tivesse de reformular a questão, como a excreveria? Partilhar a resultado com os demais elementos do grupo

f) Elaborar de forma escrita uma resposta individual a pergunta do colega a partir do ponto de vista do seu secto [articular a resposta com elementos aferidos na pesquisa documental realizada por si] ]

h) Elaborar uma resposta conjunta do grupo de "experts" para a pergunta do colega, fundamentando com tudo - que sabem e leram (colocar na vossa resposta todas as referéncias que permitem fundamentar a resposta)

28 de Maio 2018 
Anexo 2 - Guia de Pesquisa

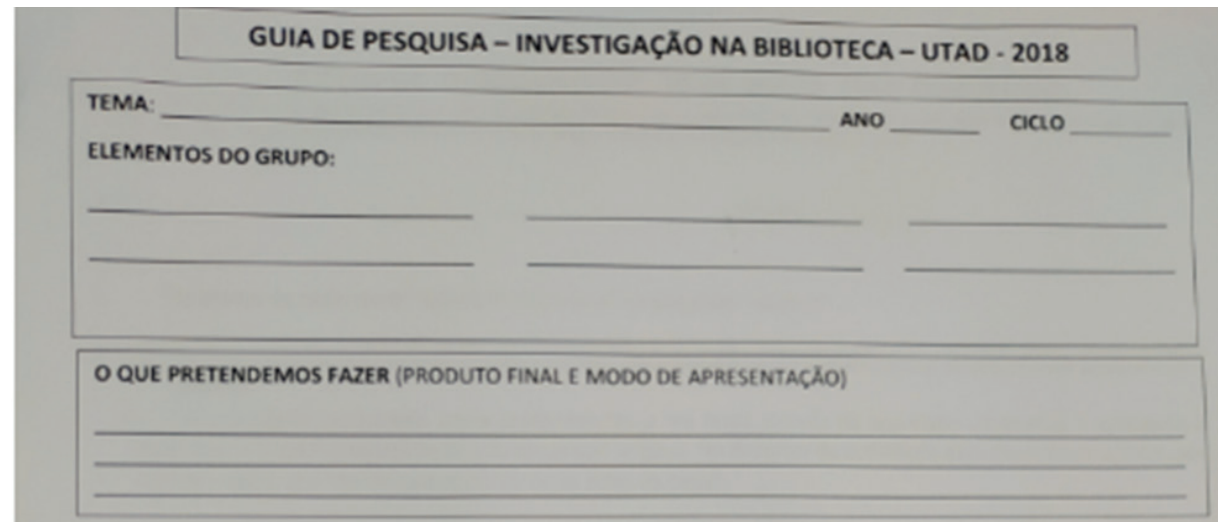

IDENTIFICAR QUATRO QUESTOES SOBRE O TEMA (e importante que as questర్́es sejam controversas, ou um problema para resolver, uma decisão a tomar, um dilema ético)

\section{SELECIONAR PALAVRAS CHAVES}

CRIAR NUVENS DE PALAVRAS (WORDART, WORDCLOUDS, ETC) I MAPAS CONCETUAIS (CMAP TOOLS)

ESCOLHER AS FONTES MAIS ADEQUADAS (aplicar os critérios de exatidåo, autoridade, objetividade, atualidade e abrangência)

REGISTO DAS FONTES (IMPRESSAS OU ELETRONICAS) - NORMAS APA

\begin{tabular}{|l|l|l|l|l|}
\hline AUTOR(ES) & DATA & TITULO & ENDERECOO ELETRONICO & DATA DA CONSULTA \\
\hline & & & & \\
\hline & & & & \\
\hline & & & & \\
\hline & & & & \\
\hline & & & & \\
\hline
\end{tabular}


Margarida Carvalho

Diretora dos Serviços de Documentação e Bibliotecas Universidade de Trás-os-Montes e Alto Douro (UTAD), Vila Real, Portugal

Email: mcarvalh@utad.pt

ORCID: 0000-0002-4762-6153

Caroline Dominguez

Professora Auxiliar Universidade de Trás-os-Montes e Alto Douro (UTAD), Vila Real, Portugal Centro de Investigação em Didática e Tecnologia na Formação de Formadores (CIDTFF), Aveiro, Portugal

Email: carold@utad.pt ORCID: 0000-0002-8486-4142

Teresa Morais

Professora

Escola Secundária S/3 São Pedro, Vila Real, Portugal

Email: morais.tm@sapo.pt ORCID: 0000-0001-6041-318X

Correspondência

Margarida Carvalho

Serviços de Documentação e Bibliotecas Universidade de Trás-os-Montes e Alto Douro

Quinta de Prados

5000-801 Vila Real

Data de submissão:

Data de avaliação:

Data de publicação: 